\title{
COMPARISON OF E-INCLUSION PREDICTION MODELS IN BLENDED LEARNING COURSES
}

\author{
Ieva Vitolina and Atis Kapenieks \\ Riga Technical University, Kalku street 1, Riga, Latvia, LV-1010
}

\begin{abstract}
E-inclusion or digital inclusion is the European Commission's effort to ensure that everybody can benefit from the opportunities provided by digital technologies. Blended learning is a convenient and affordable way to learn new digital skills. However, the problem is the low number of e-learning graduates and the even lower number of those who use the newly acquired digital skills for professional purposes. To impact blended learning course results and to improve the usage of newly acquired digital skills machine learning and data mining techniques are used. The purpose of the study is to develop and compare e-inclusion predictions models, taking into account the following conditions: 1) the prediction model must use a set of data obtained from different digital skills blended training courses; 2) the prediction model must identify as many risk learners as possible at the same time to prevent the model from targeting actual non-risk learners as at-risk. We compared seven e-inclusion prediction models for blended learning courses. The models predict digitally excluded learners using classification, clustering, linear regression algorithms, and combinations of these algorithms. We used two metrics - F1 and F2 measures for prediction models. We concluded if the aim is predicting as many students at risk as possible then the classifier ensemble method with majority voting combined with linear regression predictions is appropriate. This model predicts $98.40 \%$ of digital excluded learners, however only $58.20 \%$ of those predicted as digitally excluded are actually excluded. Combining the classifier ensemble method with the majority voting and clustering model can predict as digitally excluded $82.80 \%$ of excluded students and can predict correctly $79.60 \%$ of learners as risk learners.
\end{abstract}

\section{KEYWORDS}

e-Inclusion, Prediction, Blended Learning, Machine Learning

\section{INTRODUCTION}

E-inclusion or digital inclusion is the European Comission's effort to ensure that everybody can benefit from the opportunities provided by digital technologies. The European Commission (2020) Digital action plan states that the quality and inclusiveness of education and training systems and providing digital skills for all is of strategic importance for the EU. Recent data show that the demand for digital skills is growing (Gartner, 2020). Blended learning is a convenient and affordable way to learn new digital skills. However, the challenge is the low number of e-learning graduates and the even lower number of those who use the newly acquired digital skills for professional purposes (Eurostat, 2020). According to a CEB Global (2014) study, for the average organization, $45 \%$ of learning investments are scrap learning - learning that is delivered but not applied back on the job.

Drop out prediction and prevention have been the subject of quite a number of studies (Lu et al., 2018). To improve blended learning course results machine learning and data mining techniques are used (Sukhbaatar et al. 2018). Predictive models for blended courses are still limited. Conijn et al. (2017) analyzed 17 blended courses and concluded that the portability of the prediction models across courses is low.

No comprehensive study has been carried out to analyze how to improve digital skills training, ensuring that newly acquired skills are used meaningfully in professional life. In our previous studies, we tested several classifiers, linear regression and clustering algorithms to build e-inclusion models and to predict usage of newly acquired digital skills. The purpose of this study is to develop and compare e-inclusion prediction models, taking into account the following conditions:

1) the prediction model must use a set of data obtained from different digital skills blended training courses; 
2) the prediction model must identify as many risk learners as possible at the same time to prevent the model from targeting actual non-risk learners as at-risk.

This study will contribute to the development of predictive e-inclusion models based on data from different blended learning courses to provide information to instructors and support to students.

\section{METHODOLOGY}

We did our research based on the following steps: Data acquisition, pre-processing, attribute selection, determination of model performance metrics, application of the selected algorithms to create prediction models, comparison of models and analysis of results.

\subsection{Data Acquisition}

Data were obtained from a blended learning course "Improvement of ICT skills" and program "Modern Education of Interests" on the e-learning platform Moodle. The participants of the courses were teachers of vocational education institutions. During the courses, teachers had the opportunity to improve the digital skills needed to teach students, as well as to acquire or improve digital skills to teach students about robotics, video and mobile technologies. Additionally, data were obtained from questionnaires filled in by learners six to seven months after completing the course. Learners were asked about the usage of skills gained in the courses for their professional needs.

\subsection{Data Pre-Processing}

One of our tasks was to generalize the model so that it could be applied to different courses. Therefore, unlike our previous research, we created one data set from different courses and their topics. We merged data from the following courses and topics: Video Technology and Design course, Mobile Technologies course, Robotics course, and Improvement of ICT Skills course.

From previous studies, we have observed that predictions are inaccurate if the data set includes students who do not use the newly acquired skills because they do not have access to the required technology. Therefore, we excluded students without access to technology from the data sets. In total, the training data set included the records of 928 students who had studied different courses or topics.

\subsection{Attribute Selection}

The selection of relevant attributes affecting the prediction result was done based on Nissen (2005) knowledge management theory and previous research of factors predicting e-inclusion degree. We used seven numeric attributes for e-inclusion prediction: learner's interest in learning, learner's general digital skill level, learner's ability to learn, learner's evaluation of e-learning materials, instructor's willingness to share knowledge, learner's assessment of e-learning environment, learner's predicted use of newly learned skills.

Also, we have an attribute observed usage of newly learned skills. This attribute has 2 values: $e$-included and not e-included. We defined that the value is $e$-included if we observed that the learner uses newly learned skills. The value is not e-included, if we observed that the learner doesn't use newly acquired skills. We labeled each learner record of the dataset as e-included or not e-included. Our data set contains 435 not $e$-included learners and 493 e-included learners.

The dependent variable of the linear regression model is the numeric variable - degree of e-inclusion what is a combination of the learner's predicted and observed usage of newly acquired digital skills.

\subsection{Model Performance Metrics}

There is no consensus on which classifier performance metric is better to use (Seliya et al., 2009). In a binary classification problem the performance of the classifiers is assessed using the standard measures of recall, precision, F-measure, where: 


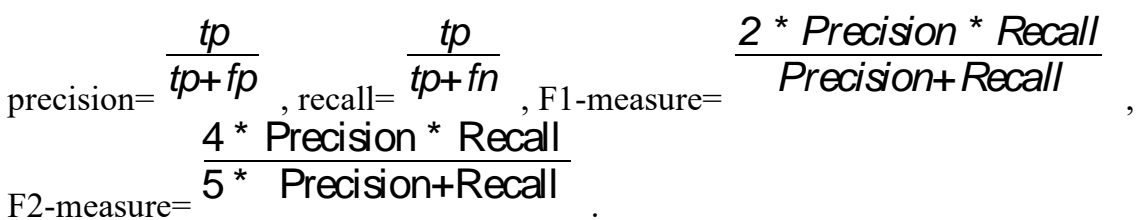

$t p$ is true positive (not e-included), tp is the amount of positive (not e-included) data that is predicted right. $t n$ is true negative (e-included $), t n$ is the amount of negative (e-included) data that is predicted right. As our goal is to identify students who are at risk of not being e-included we consider not e-included as the positive class and e-included as the negative class. $f p$ (false positive) is the amount of positive data that is predicted incorrectly. $f n$ (false negative) is the amount of negative data that is predicted incorrectly.

To determine the most appropriate model, it is essential to choose the model's performance metrics. We believe that an important indicator of the performance of the model is the recall score. Recall shows the proportion of the positive class (not e-included) instances that are correctly classified. Our secondary concern was to prevent the model from targeting actual e-included students as at-risk students. Therefore, we also decided to use precision as a metric, as well as the F measure. Precision indicates what percentage of the predictions on the positive class (not e-included) is correct.

The F1 measure is the harmonic mean between precision and recall. An e-inclusion prediction model makes sense if it covers as many students at risk as possible. Therefore, we will also use the F2 measure in our study to emphasize the importance of recall. The F2 measure combines precision and recall, putting a double emphasis on recall.

\subsection{Selection of Algorithms and Building of the Prediction Models}

For model training, we used the Waikato Environment for Knowledge Analysis (WEKA) platform (Hall et al., 2009).

\subsubsection{Prediction Model1: Classification Based E-inclusion Prediction Model}

In this study, we decided to use the ensemble method - voting to provide improvement of prediction model performance (Kumari et al., 2018). In our previous study, we tested 5 classifiers Naive Bayes, SimpleLogistic, lazy.LWL, OneR, and LMT for three different blended learning courses (Vitolina, \& Kapenieks, 2020). Our previous study has not shown that any of the classifiers perform best in all courses. However, the obtained results showed that lazy.LWL and LMT algorithms have higher scores for metrics. Therefore, we decided to use the lazy.LWL and LMT algorithms as base models and to combine them with other Naive Bayes, Simple Logistic and OneR algorithms to find out when the performance of the Vote algorithm is higher.

We were looking for a combination of base models with the highest F1 and F2 measures for the Vote algorithm (Table 1). We combined the predictions from classifiers using the majority voting approach (Dietterich, 2000). We obtained that the highest F1 measure is 0.809 when there are four base models: lazy.LWL with Random Forest, LMT, OneR, and Simple Logistic algorithms. We also determined that the F2 measure is the highest - 0.799 in the the case of three base models: lazy.LWL with Random Forest, LMT, and Simple Logistic algorithms.

Prediction Model1 is an ensemble model which combines:

1) predictions of lazy.LWL with Random Forest, LMT, OneR, and Simple Logistic algorithms using the majority vote approach in case of F1 measure.

2) predictions of lazy.LWL with Random Forest, LMT, and Simple Logistic algorithms using the majority vote approach in case of F2 measure. 
Table 1. Combinations of base models and metrics values for the Vote algorithm

\begin{tabular}{|c|c|c|c|c|c|c|}
\hline LMT & Lazy.LWL & NaiveBayes & OneR & $\begin{array}{l}\text { Simple } \\
\text { Logistic }\end{array}$ & F1 measure & F2 measure \\
\hline $\mathrm{x}$ & $\mathrm{x}$ & $\mathrm{x}$ & & & 0.801 & 0.785 \\
\hline $\mathrm{x}$ & $\mathrm{x}$ & $\mathrm{x}$ & $\mathrm{x}$ & $\mathrm{x}$ & 0.800 & 0.775 \\
\hline $\mathrm{x}$ & $\mathrm{x}$ & $\mathrm{x}$ & & $\mathrm{x}$ & 0.804 & 0.789 \\
\hline $\mathrm{x}$ & $\mathrm{x}$ & $\mathrm{x}$ & $\mathrm{x}$ & & 0.808 & 0.771 \\
\hline $\mathrm{x}$ & $\mathrm{x}$ & & & $\mathrm{x}$ & 0.807 & 0.799 \\
\hline $\mathrm{x}$ & $\mathrm{x}$ & & $\mathrm{x}$ & $\mathrm{x}$ & 0.809 & 0.784 \\
\hline $\mathrm{x}$ & $\mathrm{x}$ & & $\mathrm{x}$ & & 0.807 & 0.785 \\
\hline
\end{tabular}

\subsubsection{Prediction Model2: Clustering Based E-inclusion Prediction Model}

Our previous research has shown that cluster analysis can be used in the context of e-inclusion prediction modeling. K-means is a partitional clustering method where each object belongs in exactly one cluster (Likas et al., 2003). Euclidean distances are used to measure the dissimilarity between a data point and the cluster representatives. The K-means algorithm is used as a classifier to group data of the same class (Kumar et al., 2012). We used the K-means algorithm as a classifier to group e-included and not e-included examples together. Prediction Model2, which is based on the K-means algorithm, divides the data into 2 clusters, where each of the clusters corresponds to the e-included or not-e-included group.

\subsubsection{Prediction Model3: Linear Regression-Based E-inclusion Prediction Model}

Based on previous research, we used a multiple linear regression to predict the e-inclusion degree. The model predicted the degree of e-inclusion as a percentage of the maximum possible. To determine whether a student is e-included or not, we had to set a threshold for the percentage required for a student to be considered e-included. To set a threshold for the predicted degree of e-inclusion we compared the predicted degree of e-inclusion with the actual observation of whether or not the student uses the newly acquired skills. Figure 1 shows the change in classification metrics as the degree of e-inclusion increases. As the degree of e-inclusion increases, the precision of the model decreases, but the recall increases.

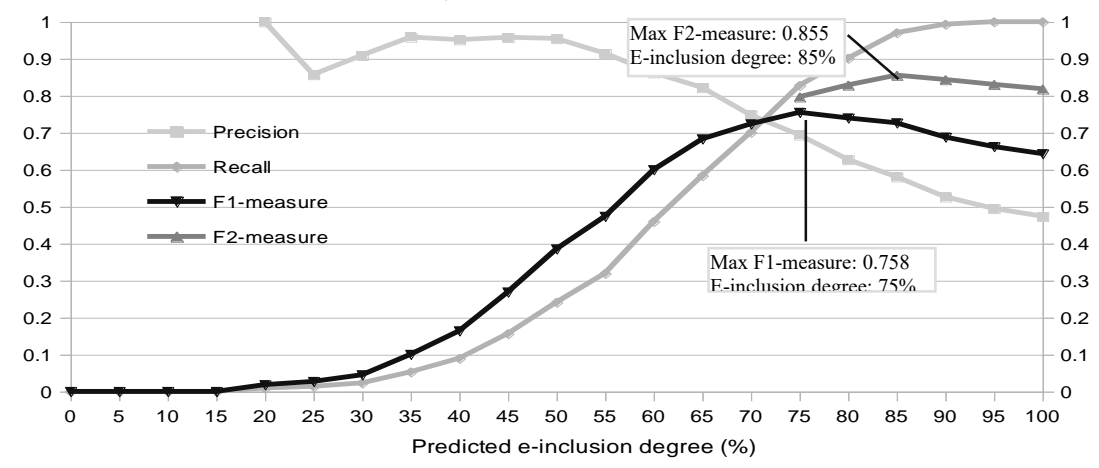

Figure 1. The change in classification metrics depending on the predicted degree of e-inclusion. The threshold of the e-inclusion degree

We established that the highest F1-measure value is when $75 \%$ of the e-inclusion rate is reached. This means that if a student is assigned an e-inclusion rate of less than $75 \%$, we assume that the student is at risk of being digitally excluded. With an e-inclusion degree $75 \%$, the recall value is 0.828 , the precision is 0.695 . The highest F2-measure value is when the e-inclusion degree has reached $85 \%$. So, if we choose the F2 measure as the determining metric, then we consider that the student is at risk if its e-inclusion degree is lower than $85 \%$. With an e-inclusion degree $85 \%$, the recall value is 0.970 , the precision is 0.580 .

Prediction Model3 is a multiple linear regression model which predicts that the learner is not e-included:

1) if the predicted e-inclusion degree is less than $75 \%$ and the F1 measure is the determining metric.

2 ) if the predicted e-inclusion degree is less than $85 \%$ and the F2 measure is the determining metric. 


\subsection{Combinations of the E-inclusion Models}

We looked for ways to improve recall rates and to reduce the number of students the model predicts to be included, but in reality, students are not e-included. We created four additional models: Model4, Model5, Model6, Model7. Fig. 2 presents rules used to combine Model1, Model2, and Model3. Model combinations are based on changing the prediction value from e-included to not e-included if the second (added) model prediction value is not e-included. In this way, we increase the number of not e-included students as a result of the prediction.

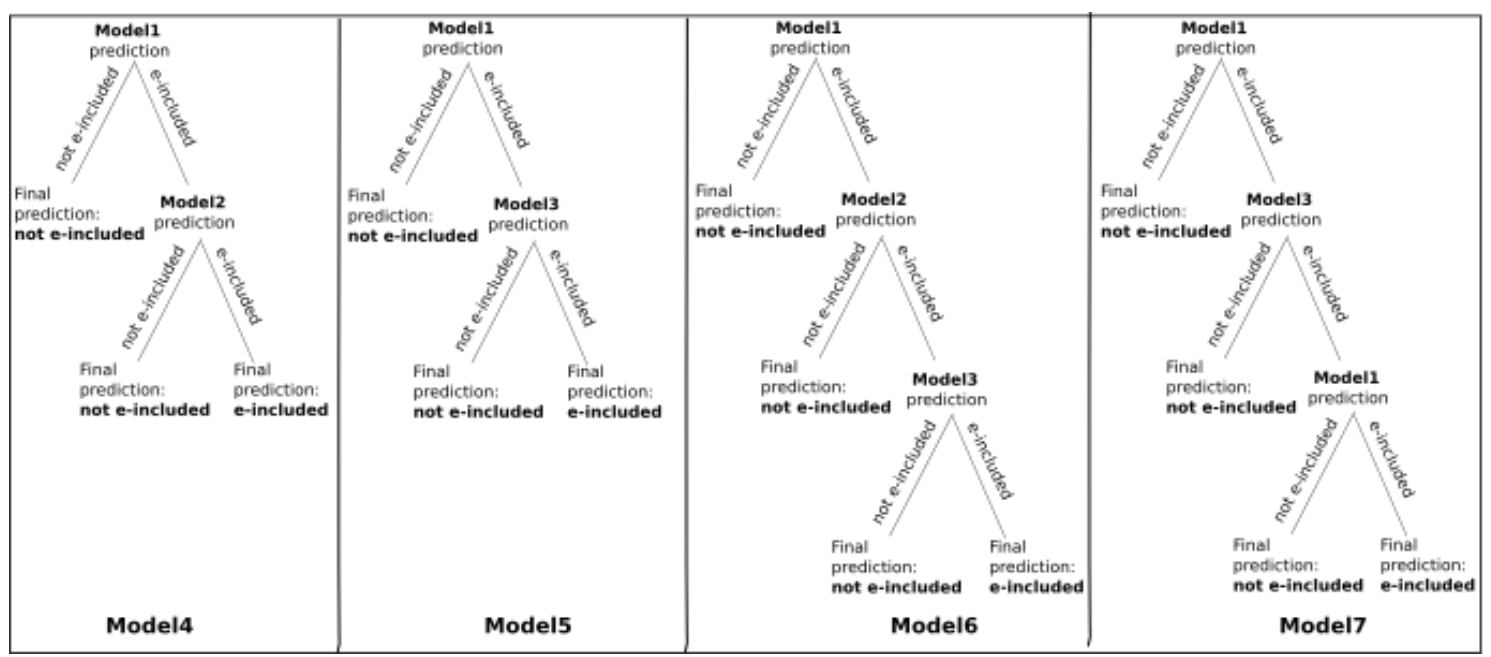

Figure 2. The rules for creating new prediction models by combining Model1, Model2, and Model3 to improve recall rates based on changing prediction value from $e$-included to not $e$-included

\subsection{Evaluation Strategy}

For e-inclusion prediction Model1, Model2, Model3 evaluation we used 10-fold cross-validation that is a widely accepted model evaluation approach in machine learning (Yadav, \& Shukla, 2016).

\section{RESULTS AND DISCUSSION}

On the WEKA platform, we trained Model1, Model2, and Model3 and then by combining these models, we got Model4, Model5, Model6, and Model7.

\subsection{Models Training Results: F1 Measure as the Main Metric}

First, we used the F1 measure value as a model performance criterion, so both recall and precision are considered equally important.

Figure 3 shows that the highest F1 measure value 0.812 is for Model4, which is obtained by combining Model1 and Model2. Model1 uses the classification algorithms lazy.LWL, LMT, OneR, and Simple Logistic, combining them with the ensemble method Vote (majority voting). Model2 is K-means clustering, where data is grouped in e-included and not e-included clusters. The recall of the Model 4 is 0.828 , the precision is 0.796. This means that $82.80 \%$ of excluded students can be predicted as risk learners, but $27.20 \%$ of excluded students will be predicted as e-included. $79.60 \%$ will be predicted correctly as risk learners, but $20.40 \%$ of e-included learners will be included in the risk group. 
The highest recall value 0.915 is for Model6 and Model7, but at the same time these models have the lowest precision value 0.692, F1 measure is 0.788 . Model6 and Model7 are the result of the combination of Model1, Model2, and Model3. Thus, these models have a combination of classifier, cluster, and linear regression predictions.

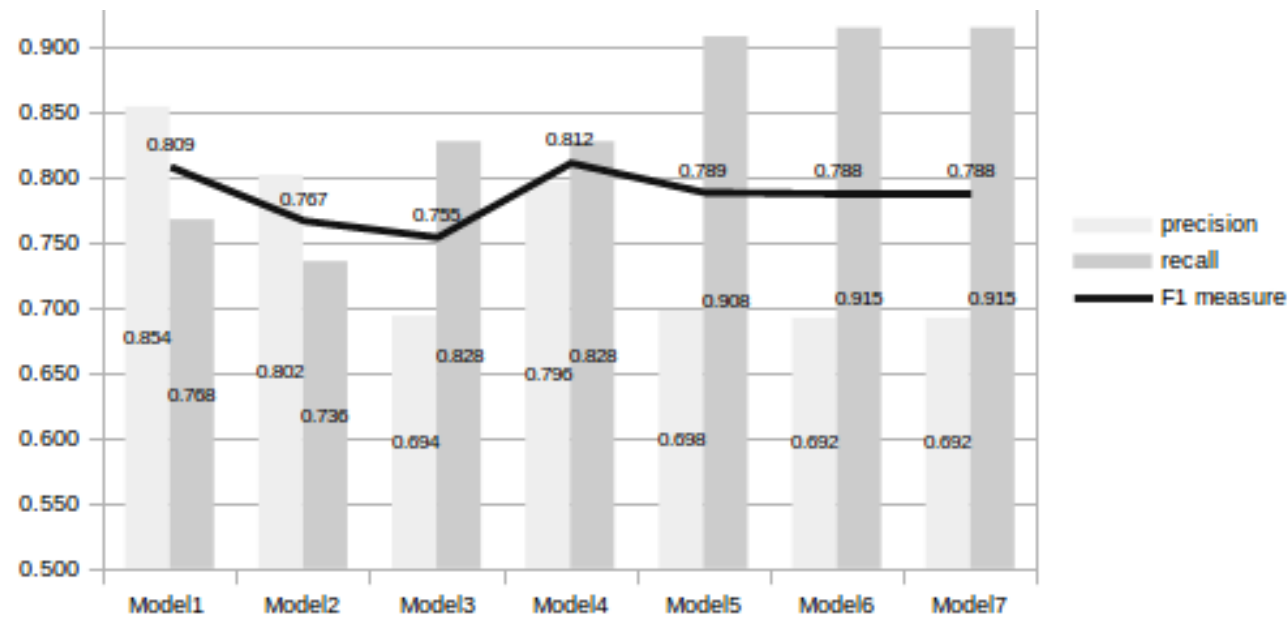

Figure 3. Results. F1 measure, precision, recall values for e-inclusion prediction models considering recall and precision with the same importance

\subsection{Models Training Results: F2 Measure as the Main Metric}

To find out which models are more suitable if recall is more important than precision, we used the F2 measure as the model metric.

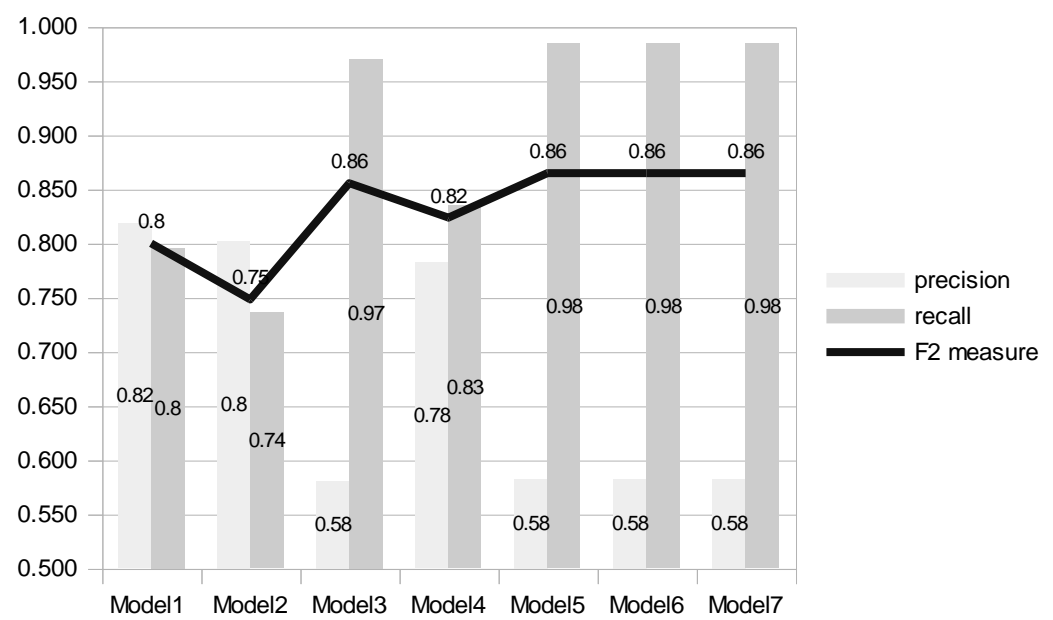

Figure 4. Results. F2 measure, precision, recall values for e-inclusion prediction models considering recall more important than precision

The highest F2 measure value 0.865 is for Model5, Model6, and Model7. Model5 is obtained by combining Modell and Model3. Modell uses the classification algorithms lazy.LWL, LMT, and Simple Logistic, combining them with the ensemble method Vote (majority voting) (Fig. 4). Model3 is a linear regression model, where the learner is e-included if its e-inclusion degree is higher than $85 \%$. Model6 and Model7 are the result of the combination of Model1, Model3 and Model3. These models have a combination of classifier, cluster, and linear regression predictions. The recall of Model5, Model6, and Model7 is 0.984, 
but precision is 0.582 . Model5, Model6, and Model7 predict $98.40 \%$ of learners at risk, however, it should be noted that only $58.20 \%$ of those predicted as learners at risk are actually excluded. $41.80 \%$ of the learners who are e-included, these models predict as at-risk students.

Model4, which combines the majority voting classification approach and clustering has an F2 measure value of 0.824 , a precision value of 0.782 , and recall value of 0.834 . For this model, the recall is lower than for Mode15, Model6, and Mode17, but the precision value is higher. Model4 predicts $83.40 \%$ of learners at risk, $21.80 \%$ of e-included learners are predicted as at-risk learners.

For Mode15, Mode16, and Model7 the accuracy is $66.60 \%$, this means that in $66.60 \%$ of cases it is possible to predict correctly. For Model4, the accuracy is $81.40 \%$ and it is higher than for Model5, Model6 and Model7.

By changing the e-inclusion threshold for the linear regression algorithm, we observed a change in the recall and precision values of the model combinations (Fig. 5). For example, if the e-inclusion threshold is $70 \%$ then the F2 measure for Model5 is 0.828 , the recall 0.851 , and the precision is 0.782 . Model6 and Model7 have such values: the F2 measure is 0.837 , the recall is 0.864 , and the precision 0.743 . In case of a threshold of $75 \% \mathrm{~F} 2$ measure for Model5 is 0.855 , the recall is 0.906 , and the precision is 0.701 . Model6 and Model 7 have an F2 measure value of 0.859 , the recall is 0.913 , and the precision is 0.696 . This means that changing the e-inclusion threshold in the prediction model allows specifying which is more important to user - precision or recall.

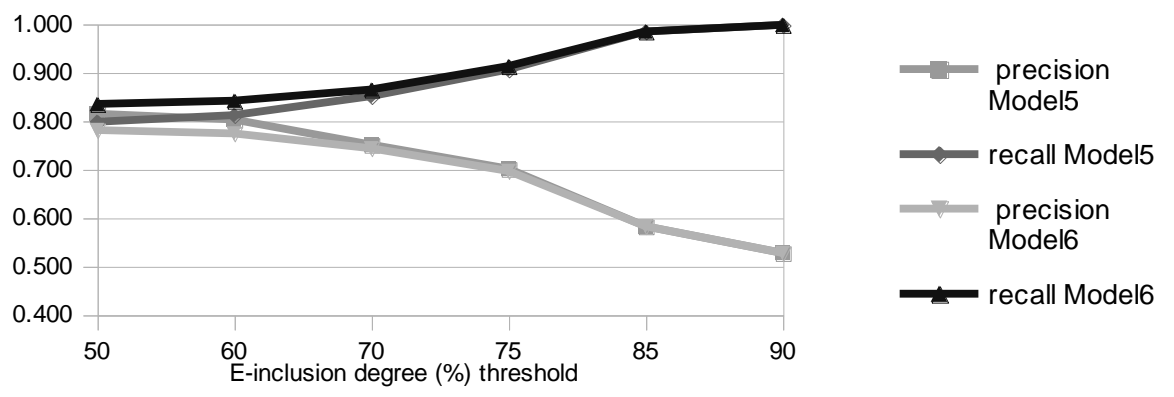

Figure 5. E-inclusion threshold and a change in the recall and precision values of the Model5 and Model6

\section{CONCLUSIONS}

In this paper we have presented and compared seven e-inclusion prediction models for blended learning courses. It should be emphasized that the models were trained with a dataset that contains records from various courses and programs. The models predict digitally excluded learners using classification, clustering, linear regression algorithms, and combinations of these algorithms. We used two metrics - F1 and F2 measures for prediction models.

It depends on the prediction goal, which model to choose as the main model for e-inclusion prediction. If it is important to identify as many students at risk as possible, then the F2 measure is an appropriate metric. If care about recall and precision is equal then the F1 measure is useful. The variety of our prediction models has the advantage of being able to choose the most appropriate model depending on your goal. However, it should be noted that if the model increases the recall value, then the precision decreases

We concluded that if the main goal is to predict as many students at risk as possible then the classifier ensemble method with majority voting combined with linear regression predictions is appropriate. This model predicts $98.40 \%$ of digital excluded learners, however, only $58.20 \%$ of those predicted as digitally excluded are actually excluded. This means that resources will be used unnecessarily to interfere in the training of $41.80 \%$ of learners. If time and financial resources are available, then a low precision value is not an obstacle.

However, if resources are limited, then probably the prediction model is more useful, where precision and recall are equally important and the F1 measure is used. We concluded that such a prediction model can be obtained by combining the classifier ensemble method with majority voting and clustering. This prediction 
model can predict as digitally excluded $82.80 \%$ of excluded students and can predict correctly $79.60 \%$ of learners as risk learners. In this case, only $20.40 \%$ of e-included learners will be predicted as digitally excluded and this is relatively less than when using the F2 measure as a metric. When using this model, it is important to understand how significant the loss is that the model did not predict $27.20 \%$ of excluded students. We concluded that change of the e-inclusion degree threshold in the linear regression model impacts the recall and precision values. Depending on the choice of metrics and the e-inclusion degree threshold, e-included or non-e-included students will be predicted more accurately.

The predictive model will be useful for both instructors and learners to identify risks in the student training process as soon as possible. Moreover, the results of this study will help us better understand how to prevent timely scrap learning in organizations whose employees learn new digital skills.

For future work we could improve the e-inclusion prediction model to be able to identify which specific factors, such as e-learning materials, e-environment, collaboration with the instructor, or others, are more important to the practical application of new digital skills. In the future, we plan to design a prototype of the e-inclusion prediction model to test it in blended learning courses.

\section{ACKNOWLEDGMENT}

This work was supported by a grant from the international European ERA-NET Project FuturICT2.0 funded under the FLAG-ERA Joint Transnational Call 2016.

\section{REFERENCES}

CEB Global. 2014. Confronting Scrap Learning, www.cebglobal.com $/ \mathrm{mtm}$

Conijn, R. et al. 2017. Predicting student performance from LMS data: A comparison of 17 blended courses using moodle LMS. In IEEE Transactions on Learning Technologies, Vol. 10, No. 1, pp. 17-29.

Dietterich, T. G. 2000. Ensemble methods in machine learning. International workshop on multiple classifier systems. Berlin, Heidelberg, Germany, pp. 1-15.

European Commision. 2020. Digital Education Action Plan 2021-2027, https:/ec.europa.eu/education/sites/education/files/document-library-docs/deap-communication-sept2020_en.pdf

Eurostat. 2020. People expanding their knowledge by learning online, https://ec.europa.eu/eurostat/web/productseurostat-news/-/EDN-20200517-1

Gartner, Inc. 2020. Lack of Skills Threatens Digital Transformation, https://www.gartner.com/smarterwithgartner/lackof-skills-threatens-digital-transformation/

Hall, M. et al. 2009. The WEKA data mining software: an update. In ACM SIGKDD explorations newsletter, Vol. 11, No. 1, pp.10-18.

Kumar, A. et al. 2012. Modeling using K-means clustering algorithm. 1st International Conference on Recent Advances in Information Technology (RAIT). Dhanbad, India, pp. 554-558, doi: 10.1109/RAIT.2012.6194588

Kumari, P. et al. 2018. An efficient use of ensemble methods to predict students academic performance. 2018 4th International Conference on Recent Advances in Information Technology (RAIT). Dhanbad, India, pp. 1-6.

Likas, A. et al. 2003. The global k-means clustering algorithm. Pattern recognition, Vol. 36, No. 2, pp. 451-461.

$\mathrm{Lu}, \mathrm{O}$. et al. (2018). Applying learning analytics for the early prediction of Students' academic performance in blended learning. Journal of Educational Technology \& Society, Vol. 21, No. 2, pp. 220-232.

Nissen, M. E. (Ed.). 2005. Harnessing Knowledge Dynamics: Principled Organizational Knowing \& Learning: Principled Organizational Knowing \& Learning. IGI Global.

Romero, C. and Ventura, S. 2019. Guest Editorial: Special Issue on Early Prediction and Supporting of Learning Performance. In IEEE Transactions on Learning Technologies, Vol. 12, No. 2, pp. 145-147.

Seliya, N. et al. 2009. A study on the relationships of classifier performance metrics. In 21st IEEE International Conference on Tools with Artificial Intelligence. Newark, NJ, pp. 59-66.

Sukhbaatar, O. et al. 2018. Mining Educational Data to Predict Academic Dropouts: a Case Study in Blended Learning Course. In TENCON 2018-2018 IEEE Region 10 Conference, pp. 2205-2208.

Vitolina, I., Kapenieks A. (2020). E-inclusion Prediction Modelling in Blended Learning Courses (accepted paper), 23rd International Conference on Interactive Collaborative Learning 